\title{
Identifying Opinion Leaders in Time-Dependent Commercial Social Networks
}

\author{
Antonio P. Volpentesta and Alberto M. Felicetti \\ Department of Electronics, Computer Science and Systems, \\ University of Calabria, via P. Bucci, 42\C, 87036 Rende (CS), Italy \\ \{volpentesta, afelicetti\} adeis.unical.it
}

\begin{abstract}
The increasing amount of information flowing through commercial social networks offers clear advantages for companies who can take a valuable feedback from community actions. In particular, the identification of influential users in on-line social network can support companies in designing and targeting marketing campaigns, as influential gate-keepers and diffusers of information can ignite epidemics through word-of-mouth. In this paper, we model a time-dependent commercial social network as a time-varying weighted directed graph. Moreover, we propose an approach to determine opinion leaders and their contributions to a temporal business value, by taking into account behavioural and structural aspects of the commercial social network.
\end{abstract}

Keywords: Opinion leaders, Social Networks, Social Network Centrality, Viral Marketing.

\section{Introduction}

Nowadays, internet based technologies, and in particular Web 2.0 technologies, provide a multitude of platforms, such as blogs, wikis, social networks and forums where users can disseminate an abundance of information on personal experiences and opinions about products and manufacturers [1]. The advent of participatory web enables users to produce and share on-line content, radically changing the traditional communication paradigms and turning the former mass information consumers to the present information producers [2]. Hence, from a company point of view, the consumer role shifts from a pure consumer of products to a partner in the value creation process [3].

There are several motivations that drive users to share online-content within their own network of social relationships:

- The need to be part of a group, as well as to establish and maintain a certain number of social relationships [4].

- The need to affirm their own individuality: users, as consumers, "identify with products or brands to the degree that the product actually becomes part of the consumer's extended self" and they "communicate something important about themselves to others", [5]. 
- Curiosity and fun: users are encouraged to share entertainment based contents, which attract their curiosity by leveraging on their passions [6].

It is widely accepted that word-of-mouth communication, both in real life and in online social networks, plays an important role in shaping users' attitudes and behavior [7]. Daily, our own decisions are heavily influenced by the opinions of the people around us and this influence increases with the strength of the relationship. In fact, seeing actions performed by our friends may make us curious and may sometimes tempt us to perform those actions ourselves, [8]. According to [2] and [9], the majority of people prefer consulting family, friends or colleagues over traditional advertising before buying a new product or experiencing a new service. In other words, before people make decisions, they talk, and they listen to other's experience, opinions, and suggestions.

Actually, when people perform an action, they may be influenced by what they have heard of it outside of the online social network (and have decided it is worthwhile) or they may be genuinely influenced by seeing their social contacts performing that action [10]. In what follows, when we talk about influence, we only refer to on-line genuine influence. Even in this case, users of a social networks, do not exert the same level of influence over the other participants. In fact, community members differ widely in terms of the frequency, volume, type, and quality of digital content generated and consumed [11]. Besides, some community members are more influent than other users. Through their own opinions, they guide perceptions and actions of others with respect to specific topics (eg. politics, sports, culture but also products / services / brands).

On one hand, a user may have practical and emotional benefits when participating in online discussions and content sharing processes; on the other hand it appears evident how these conversations have profound commercial implications as well [9]. In fact, in recent years consumers have shown a growing resistance to traditional forms of advertising like television commercials or newspaper [12], becoming more aware of their needs and expectations. Therefore, in addition to relying upon traditional media, many companies advertise their products and services, through new marketing strategies (e.g. "Viral Marketing") that leverage Web 2.0 technologies .

The Viral Marketing concept is based on promoting products and services through the exploitation of internet word-of-mouth, in order to achieve widespread promotional message among users [13]. The message diffusion follows an exponential model, similarly to a virus within a population.

The "social environment" enables mechanisms of interaction, cooperation and "social experience" among users, and between consumers and companies who can take a valuable feedback from the overall community. According to this perspective, the identification of most influential users (also called "opinion leaders") in a web community is a valuable problem to be studied.

Such a problem is still more important in the case of a time-dependent commercial social network (CSN). A CSN is a social network "designed to support business transaction and to build a trust between an individual and a brand, which relies on opinion of product, ideas to make the product better, enabling customers to participate 
with the brands in promoting development, service delivery and a better customer experience" [14].

In a CSN, opinion leaders are individuals who, through their actions and opinions, guide the perceptions of other CSN members towards products or services provided by some companies. In particular, each of them exerts a certain degree of influence on the behavior of customers in the various stages of their purchasing process. These influences may vary with time and have a relevant impact on the CSN business value. Broadly speaking, CSN business value comprises all intangible benefits deriving from social interactions that occur within a CSN in given interval times.

In this paper, we model the influence relationship in a CSN as a time-varying weighted direct graph, and we propose an approach to determine opinion leaders and their contributions to the business value of a time-dependent CSN.

\section{Related Works}

In the last decade, social platforms have radically changed the way users interact and share information. Social networks are usually modeled through a mathematical formalism based on graph theory, where the nodes represent individuals and edges (or arcs) represent the relationships and interactions between individuals [15]. Identifying influencer people through social network analysis (SNA) is gaining prominence in many application areas [16].

In order to identify the roles of individuals in the network, SNA evaluates the importance (also called centrality) of actors in the network as well as analyzes their behaviors and their interactions. In particular, two types of network analysis are studied in literature, [17]: Structural Analysis (which concentrates on measuring centrality taking into account the structure of a network) and Behavioral Analysis (which focuses on the interactions between users rather than the structure of the network, identifying followers who propagate or share contents, and users who are engaged in conversations).

From a structural perspective, several authors have proposed different ways to measure the "importance" of a node in a network: Closeness centrality and Graph centrality [18] are based on the distances with the rest of nodes, while Betweeness centrality and Stress centrality [19] emphasize the medium mediating between a pair of nodes. Another centrality measure that is often used in network analysis is eigenvector centrality [20]. Eigenvector centrality analysis is based on the idea that a node is "more central" if it is in relation with nodes that are themselves central, so the centrality of a node does not only depend on the number of its adjacent nodes, but also on their value of centrality.

More specifically, several studies have highlighted how the structure of a social network can affect the dynamics of user influence in social activities. In [21] and [22] authors measure influence in terms of messages propagation and forwarding activities. Cha et al. [23] use Spearman's rank correlation coefficient in order to measure users' influence in Twitter, while in [24] Rad and Benyoucef study influence in terms of link strength and incoming/ outgoing activities for each node in the 
network. In [25], two basic diffusion models are investigated: Linear Thresholds Model and Independent cascade Model. Kundu et al. [26] propose an independent cascade model and a centrality measure in order to find top $k$ influential nodes in large scale directed social networks. In [2] authors propose a frequent pattern mining approach to discover leaders in social networks, studying the propagation of their "influence", while in [11] authors develop an approach to determine "influencing users" based on a nonstandard form of Bayesian shrinkage.

The implicit assumption that underpins the above mentioned researches is that the social network is substantially static, i.e. it has time invariance. As a consequence, most of the proposed approaches are not always well suited to evolving social networks, whose topology changes either at discrete time points or continuously over time [27], [28]. This is the reason why new approaches for the identification of opinion leaders in complex time-dependent networks have been investigated [29], [30]. However, such approaches lack in considering the relevance of the business value impact factor in ranking opinion leaders.

\section{An Approach for Opinion Leaders Identification}

The identification of opinion leaders and their contribution to a CSN business value constitute an important problem for the CSN company and also all the stakeholders. In a CSN, users are considered to be "potential consumers" of some products or services, and their social interactions are revealed by analyzing their actions on timevarying digital objects on the CSN platform.

Three main assumptions underpin our approach:

1. Opinion leaders centrality can be measured by taking into account users' actions that are performed on digital objects on the CSN platform, during time intervals in which the CSN is observed.

2. The CSN business value can be decomposed in chunks, each of which is due to actions that are performed on a certain digital object in a given time interval.

3. The higher is a business value chunk, referred to an object and to an interval time, the more valuable is the centrality of an opinion leader in the CSN restricted to actions performed on that object in that time interval.

Under these assumptions we propose an approach to rank opinion leaders with respect to both their influence degree in a time-dependent CSN and a decomposition of the CSN business value.

Such approach is essentially based on a measure of the dynamic centrality of nodes in a time-varying weighted directed graph. In such graph, the weight associated to an $\operatorname{arc}(x, y)$ represents a measure of influence of $\mathrm{x}$ on $\mathrm{y}$, and varies at discrete times.

Main steps in our approach are the following:

1. Model the evolution of the influence relationship in a CSN as sequences of weighted directed graphs $G^{l}(o), G^{2}(o), \ldots, G^{k}(o)$, where the generic $G^{i}(o)$ is the model of influence relationship derived from actions on an object $o$ in time interval $\mathrm{T}^{\mathrm{i}}$. 
2. Compute the eigenvector centrality of any vertex in any graph $G^{i}(o)$.

3. Compute temporal (business) valued centrality in the CSN.

\subsection{Modeling Influence Relationship in a CSN}

In order to present the model we need to clarify some basic terms: temporal object, user and action.

An object consists of a pair (data, metadata), called object state, and an identifier, called object identifier. For instance, an object may represent a post (containing text, weblinks or digital media) published on a SN platform, a post with some comments, a list of members in a thematic group, and so on.

Depending on the type of the object, a certain number of types of actions can be performed on the object state. An action is what determines the transition from one object state to another (a special action is the "object creation" which determines the transition from the null object state to an initial object state). For instance, if the object represents a post, a user may view this post, may add a comment, may rate it (i.e. "I like" or other rate scale), or may share the post. Here, a user is an entity (individual, organization, ...) identified by an account that allows him/her to access the SN platform and to perform some type of actions on an object state.

A temporal ( or equivalently, time-varying) object is defined by an object identifier and a temporal sequence of object states, where a generic element (except the first one) is obtained by performing a suitable action on the previous one. A temporal object is used to represent the object evolution that has been happened on a $\mathrm{SN}$ platform in a certain interval time.

Formally, given an object identifier $o$, we consider the temporal object $s(o)=\left(\mathrm{s}_{l}\right.$, $s_{2}, \ldots, s_{n}$ ), where $\mathrm{s}_{1}$ represents the null object state, $s_{2}$ the initial object state, and the generic element $s_{\mathrm{i}}, \mathrm{i}>1$, represents the object state at time $t_{i}$, after a user $u_{i-1}$ has performed an action $a_{i-1}$ on the object state $\mathrm{s}_{i-1}$. The four sequences $s(o), a(o)=\left(a_{l}\right.$, $\left.a_{2}, \ldots, a_{n-1}\right), \mathrm{u}(o)=\left(u_{1}, u_{2}, \ldots, u_{n-1}\right), t(o)=\left(t_{1}, t_{2}, \ldots, t_{n-1}\right)$, describe the evolution story of the object identified by $o$ in terms of what, how, who and when.

Given a set $N$ of users of the SN platform, the influence relationship, due to actions on temporal object $s(o)$, can be modeled by a weighted directed $G(o)=$ $\{N, E(o), w(o)\}$, where:

- $E(o)=\left\{(x, y) \in N \times N: C(o)_{x, y} \neq \emptyset\right\}$, where $C(o)_{x, y}=\{(\mathrm{i}, \mathrm{j}) \in\{1, \ldots, \mathrm{n}-2\} \times$ $\left.\{2, \ldots, \mathrm{n}-1\}: x=u_{i}, y=u_{j}, i<j,\right\}$, for $x, y \in N, x \neq y$, and $C(o)_{x, x}=\emptyset, x \in N$.

In other words, a user $y$ performing an action on $o$ at a certain time is influenced by another user $x$ that has previously performed an action on the same object.

- $w(o): E(o) \rightarrow \mathbb{N} \cdot w(o)_{(x, y)}$ is a nonnegative integer that represents the weight of the influence exerted by $x$ on $y$, with respect to actions performed on a given object $o$. In order to specify $w(o)$ we need to introduce some further notations:

- Let $A T_{1}, A T_{2, \ldots,} A T_{k}$ the types of action that can be performed on $o$. 
- Let $\mathrm{D}=(\mathrm{d} i j), \mathrm{i}, \mathrm{j}=1, \ldots \mathrm{k}$, a nonnegative integer matrix, where $\mathrm{d}_{\mathrm{ij}}$ is the degree of influence that is exerted on a user performing an action of type $A T_{j}$, by a user who has previously performed an action of type $A T_{i}$.

- Given $(i, j) \in C(o)_{x, y}$, let $\mathrm{AT}_{h_{i}}$ be the action type of $a_{i}$, and $\mathrm{AT}_{l_{j}}$ the action type of $a_{j}$

The weight function $w(o)$ is defined as follows:

$$
w(o)_{x, y}=\sum_{(i, j) \in C(o)_{x, y}} \mathrm{~d}_{h_{i} l_{j}}
$$

\subsection{Centrality in an Influence Graph}

In order to determine centrality vector in an influence graph, we can use the eigenvector centrality, based on the he well known mutually reinforcing relationship assumption [31]: "a node is important if it is connected with other important nodes.

Eigenvector centrality, also called rank prestige [32] is a measure in which the centralities or statuses of positions are recursively related to the centralities or statuses of the positions to which they are connected. We assume that if a user is recognized as influential by someone seen in turn as influential by others, this may contribute to the influence measure of the first one.

Let $F=\left(f_{i j}\right)$ be an adjacency matrix of an influence graph, where $f_{i j}$ represents a measure of the influence exerted by a user $i$ on a user $j$, and let $x$ be a vector of centrality scores. Importance of a generic user $i$ is proportional to the influence exerted on other users. Numerically, it is natural to express this mutually reinforcing relationship as follows:

$$
x_{i}=c\left(f_{i 1} x_{1}+f_{i 2} x_{2}+\cdots+f_{i n} x_{n}\right)
$$

In matrix notation with $\mathrm{x}=\left(x_{1}, x_{2}, \ldots, x_{n}\right)$ this yields

$$
F^{T} X=\lambda x \quad \text { where } \lambda=1 / c
$$

Standards results of linear algebra lead to state that (2) is a solvable system of equations. If $F$ is an $n \times n$ matrix, Eq. (2) has $n$ solutions corresponding to $n$ values of $\lambda$. More precisely, a solution is given by setting $\lambda=\lambda^{*}$, the dominant $F^{T}$ eigenvalue, and $x=x^{*}$, a nonnegative eigenvector of $F^{T}$ in the eigenspace associated with $\lambda^{*}$. A normalization of $x^{*}$ gives a measure of the eigenvector-centrality of the nodes in an influence graph. The effect that different normalizations have on the interpretation of eigenvector-centrality within a graph is investigated in [33]. In order to calculate eigenvector centrality many algorithms have proposed in literature. Most of them are based on adaptations of the Hits (Hyperlink-Induced Topics Search) algorithm, introduced by Kleinberg, [32], [34].

\subsection{Temporal Valued Centrality in a CSN}

In order to define and compute the temporal (business) valued centrality we consider a sequence of time intervals $T^{1}, T^{2}, \ldots T^{k}$, and a given set $O$ of objects. Let us 
consider the subsets $C^{i}(o)_{\mathrm{x}, \mathrm{y}}=\left\{(\mathrm{p}, \mathrm{q}) \in C(o)_{x, y}: t_{p}, t_{q} \in T^{i}\right\}, i=1,2 \ldots, k$. The $i$-th footprint $G^{i}(o)=\left\{N, E^{i}(o), w^{i}(o)\right\}$ of the graph $\mathrm{G}(o)=\{N, E(o), w(o)\}$, is defined by:

- $E^{i}(o)=\left\{(x, y) \in E(o): C^{i}(o)_{x, y} \neq \emptyset\right\}$

- $\quad w^{i}(o): E^{i}(o) \rightarrow \mathbb{N} . w^{i}(x, y)$ represents the weight of the influence exerted by $x$ on $y$ in a given time interval $T^{i}$, with respect to actions performed on an object $o$.

$$
w^{i}(o)_{x, y}=\sum_{(p, q) \in C^{i}(o)_{x, y}} \mathrm{~d}_{h_{p} l_{q}},
$$

Let $e v c^{i}(o)_{x}, x \in N$, be the eigenvector centrality of $x$ in the i-th footprint $G^{i}(o)$. Let $v^{i}(o)$ be the nonnegative real number that represents the part of CSN business value $v$, due to social interactions occurring when actions are performed on $o$ in $T$.

Set $\lambda^{i}(o)=\frac{v^{i}(o)}{v}$. The temporal (business) valued centrality of $x$, is defined by:

$$
t v c_{x}=\sum_{o \in O} \sum_{i=1}^{k} \lambda^{i}(o) * e v c^{i}(o)_{x}
$$

\subsection{An Example}

Let $\mathrm{T}^{1}=[1,10), \mathrm{T}^{2}=[10,20), \mathrm{T}^{3}=[20,30) ; N=\{x, y, v, z\}, O=\{o\}$, where $o$ is the identifier of a post that evolves as time varies.

The types of action that can be performed on $o$ are:

- $\quad A T_{1}=$ create a post.

- $A T_{2}=$ view a post.

- $A T_{3}=$ share a post.

- $A T_{4}=$ add a comment to a post.

- $A T_{5}=$ rate a post (i.e. "this post was helpful to me", "I like”)

In our example, we may consider the following entries of matrix D:

Table 1. Example of action influence matrix

\begin{tabular}{|c|c|c|c|c|c|}
\cline { 2 - 6 } \multicolumn{1}{c|}{} & $\mathrm{AT}_{1}$ & $\mathrm{AT}_{2}$ & $\mathrm{AT}_{3}$ & $\mathrm{AT}_{4}$ & $\mathrm{AT}_{5}$ \\
\hline $\mathrm{AT}_{1}$ & 0 & $5 / 100$ & $18 / 100$ & $18 / 100$ & $10 / 100$ \\
\hline $\mathrm{AT}_{2}$ & 0 & 0 & 0 & 0 & 0 \\
\hline $\mathrm{AT}_{3}$ & 0 & $1 / 100$ & $4 / 100$ & $6 / 100$ & $4 / 100$ \\
\hline $\mathrm{AT}_{4}$ & 0 & $3 / 100$ & $6 / 100$ & $10 / 100$ & $6 / 100$ \\
\hline $\mathrm{AT}_{5}$ & 0 & $1 / 100$ & $2 / 100$ & $4 / 100$ & $2 / 100$ \\
\hline
\end{tabular}

and the following sequences describing temporal evolution of object $o$.

Table 2. Example of temporal evolution of an object $o$

\begin{tabular}{|c|c|c|c|c|c|c|c|c|c|c|c|c|c|}
\hline$s(o)$ & $\mathrm{s}_{1}$ & $\mathrm{~s}_{2}$ & $\mathrm{~s}_{3}$ & $\mathrm{~s}_{4}$ & $\mathrm{~s}_{5}$ & $\mathrm{~s}_{6}$ & $\mathrm{~s}_{7}$ & $\mathrm{~s}_{7}$ & $\mathrm{~s}_{8}$ & $\mathrm{~s}_{9}$ & $\mathrm{~s}_{10}$ & $\mathrm{~s}_{11}$ & $\mathrm{~s}_{12}$ \\
\hline$a(o)$ & $a_{1=} A T_{1}$ & $a_{2=} A T_{2}$ & $a_{3=} A T_{4}$ & $a_{4=} A T_{2}$ & $a_{5=} A T_{3}$ & $a_{6=} A T_{4}$ & $a_{7=} A T_{2}$ & $a_{8=} A T_{5}$ & $a_{9=} A T_{4}$ & $A_{1=} A T_{4}$ & $a_{11} A T_{3}$ & $a_{12=} A T_{4}$ & $a_{12=} A T_{4}$ \\
\hline$u(o)$ & $u_{1}=x$ & $u_{2}=z$ & $u_{3}=z$ & $u_{4}=v$ & $u_{5}=v$ & $u_{6}=x$ & $u_{7}=y$ & $u_{8}=y$ & $u_{9}=x$ & $u_{10}=y$ & $u_{11}=z$ & $u_{12}=v$ & $u_{13}=z$ \\
\hline$t(o)$ & $\mathrm{t}_{1}=1$ & $\mathrm{t}_{2}=3$ & $\mathrm{t}_{3}=7$ & $\mathrm{t}_{4}=8$ & $\mathrm{t}_{5}=10$ & $\mathrm{t}_{6}=12$ & $\mathrm{t}_{7}=13$ & $\mathrm{t}_{8}=16$ & $\mathrm{t}_{9}=18$ & $\mathrm{t}_{10}=21$ & $\mathrm{t}_{11}=25$ & $\mathrm{t}_{12}=26$ & $\mathrm{t}_{12}=28$ \\
\hline
\end{tabular}

The following footprints represent the influence relationship evolution: 


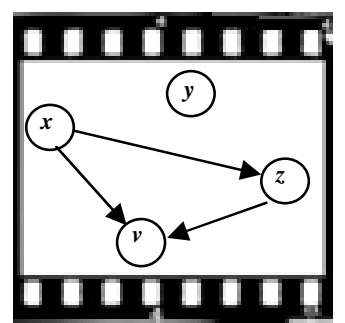

$G^{l}(o)$

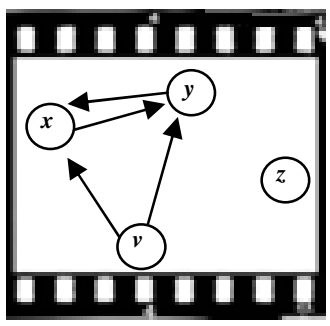

$G^{2}(o)$

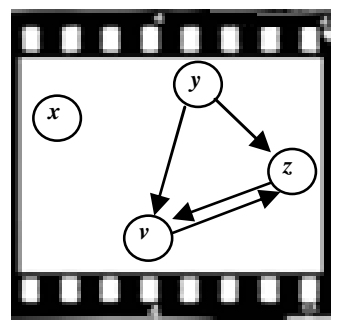

$G^{3}(o)$

Fig. 1. i-th"Footprints" of the overall weighted directed G(o)

The overall graph $\mathrm{G}(\mathrm{o})$ represents the influence relationship exerted through actions performed on the object $o$, in $\mathrm{T}=\mathrm{T}^{1} \cup \mathrm{T}^{2} \mathrm{U} \mathrm{T}^{3}$

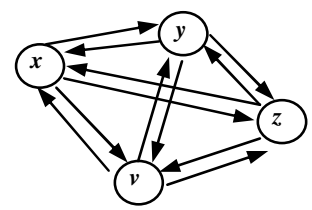

Fig. 2. the overall weighted directed G(o)

Alternative 1. ("static" eigenvector centrality). The weighted adjacency matrix of $\mathrm{G}(\mathrm{o})$ is:

\begin{tabular}{|c|c|c|c|c|}
\cline { 2 - 5 } \multicolumn{1}{c|}{$W$} & $x$ & $y$ & $v$ & $z$ \\
\hline$x$ & 0 & 0,15 & 0,51 & 0,39 \\
\hline$y$ & 0,04 & 0 & 0,14 & 0,22 \\
\hline$v$ & 0,16 & 0,3 & 0 & 0,42 \\
\hline$z$ & 0,1 & 0,19 & 0,25 & 0 \\
\hline
\end{tabular}

The eigenvector centrality (with respect a sum normalization) is:

\begin{tabular}{|c|c|c|c|c|}
\cline { 2 - 5 } \multicolumn{1}{c|}{} & $X$ & $y$ & $V$ & $z$ \\
\hline evc & 0,402139 & 0,1631016 & 0,2572193 & 0,1775401 \\
\hline
\end{tabular}

Alternative 2. (Temporal valued centrality). Let $\lambda^{1}(o)=0,2 ; \lambda^{2}(o)=0,5 ; \lambda^{3}(o)=$ 0,3 .The weighted adjacency matrices of the three footprints of $G(0)$ are:

\begin{tabular}{|c|c|c|c|c|}
\cline { 2 - 5 }$W^{l}$ & $x$ & $Y$ & $v$ & $z$ \\
\hline$x$ & 0 & 0 & 0,05 & 0,23 \\
\hline$y$ & 0 & 0 & 0 & 0 \\
\hline$v$ & 0 & 0 & 0 & 0 \\
\hline$z$ & 0 & 0 & 0,06 & 0 \\
\hline
\end{tabular}

\begin{tabular}{|c|c|c|c|c|}
\cline { 2 - 5 } \multicolumn{1}{c|}{$W^{2}$} & $x$ & $y$ & $v$ & $z$ \\
\hline$x$ & 0 & 0,09 & 0 & 0 \\
\hline$y$ & 0,04 & 0 & 0 & 0 \\
\hline$v$ & 0,12 & 0,05 & 0 & 0 \\
\hline$z$ & 0 & 0 & 0 & 0 \\
\hline
\end{tabular}

\begin{tabular}{|c|c|c|c|c|}
\cline { 2 - 5 } \multicolumn{1}{c|}{$W^{3}$} & $x$ & $y$ & $v$ & $z$ \\
\hline$x$ & 0 & 0 & 0 & 0 \\
\hline$y$ & 0 & 0 & 0,1 & 0,16 \\
\hline$v$ & 0 & 0 & 0 & 0,1 \\
\hline$z$ & 0 & 0 & 0,06 & 0 \\
\hline
\end{tabular}


The temporal valued centrality (with respect a sum normalization) is:

\begin{tabular}{|c|c|c|c|c|}
\cline { 2 - 5 } \multicolumn{1}{c|}{} & $x$ & $y$ & $v$ & $z$ \\
\hline tvc & 0,318395 & 0,32692 & 0,317751 & 0,036935 \\
\hline
\end{tabular}

In alternative 1 , the temporal evolution of the network has not been taken into account. As a consequence, the user $x$ is regarded as the "most important" opinion leader, even if he/she has been active only in the initial phase of temporal evolution of the object $o$.On the contrary, our approach (in alternative 2) gives the temporal (business) valued centrality of network users; $x, y$ and $v$ are almost equally ranked as opinion leaders. This is due to the fact that we have considered different CSN business values chinks, for each time interval, and the users have participated in a discontinuous way to the "CSN's life".

\section{Conclusions and Future Works}

Social networks have a strong impact on the way users interact and share information. The individuation of influential people can help companies, advertisers and marketing professionals in designing more effective campaigns. In particular, online opinion leaders should be targeted as a high priority group in viral marketing campaigns, as they can ignite epidemics like influential gate-keepers and diffusers of information on the Internet [35].

In this paper we have introduced an approach aimed to determine opinion leaders and their contributions to the business value of a time-dependent commercial social network (CSN). More specifically, our approach takes into account both behavioural aspects (actions users perform on the CSN platform in different time intervals) and structural aspects (eigenvector centrality) of a CSN. Future steps should be addressed to the validation and experimentation of the underlying model, as well as to further extensions where trust aspects of the relationship between users are considered.

Of course, individuals may interact with people inside and outside their CSN. A limitation of the model lies in that it does not consider interactions occurring through other web platforms or digital media (instant messaging, SMS, e-mails, and so on) as well as offline interactions. Besides, present features of today's advanced platforms of social commerce make also available data on the influence that opinion leaders exert on customers in persuading them and conditioning their purchasing behavior. These remarks suggest new research directions aimed to overcome current shortages of the approach presented in our work.

\section{References}

1. Bodendorf, F., Kaiser, C.: Detecting Opinion Leaders and Trends in Online Communities. In: Fourth International Conference on Digital Society (2010)

2. Agarwal, N., Liu, H., Tang, L., Yu, P.S.: Identifying the Influential Bloggers in a Community. In: WSDM 2008, Palo Alto, California, USA (2008) 
3. Romero, D., Molina, A.: Value Co-creation and Co-innovation: Linking Networked Organisations and Customer Communities. In: Camarinha-Matos, L.M., Paraskakis, I., Afsarmanesh, H. (eds.) PRO-VE 2009. IFIP AICT, vol. 307, pp. 401-412. Springer, Heidelberg (2009)

4. Ho, J.Y.C., Dempsey, M.: Viral marketing: Motivations to forward online content. Journal of Business Research 63, 1000-1006 (2010)

5. Chung, C., Darke, P.: The consumer as advocate: self-relevance, culture, and word-ofmouth. Marketing Letters 17(4), 269-279 (2006)

6. Ramaswamy, V.: Co-creating value through customers' experiences: the Nike case. Strategy \& Leadership 36(5), 9-14 (2008)

7. Vilpponen, A., Winter, S., Sundqvist, S.: Electronic word-of-mouth in online environments: exploring referral network structure and adoption behavior. Journal of Interactive Advertising 6(2), 63-77 (2006)

8. Kim, Y.A., Srivastava, J.: Impact of Social Influence in E-Commerce Decision Making. In: Proceedings of ICEC 2007, Minneapolis, Minnesota, USA, August 19-22 (2007)

9. Riegner, C.: Word of Mouth on the Web: The Impact of Web 2.0 on Consumer Purchase Decisions. Journal of Advertising Research (2007)

10. Goyal, A., Bonchi, F., Lakshmanan, L.V.S.: Learning Influence Probabilities In Social Networks. In: WSDM 2010, New York City, New York, USA (2010)

11. Trusov, M., Bodapati, A.V., Bucklin, R.E.: Determining Influential Users in Internet Social Networks. Journal of Marketing Research XLVII, 643-658 (2010)

12. Leskovec, J., Adamic, L.A., Huberman, B.A.: The dynamics of viral marketing. ACM Transactions on the Web 1, 1 (2007)

13. Dobelea, A., Tolemanb, D., Beverland, M.: Controlled infection! Spreading the brand message through viral marketing. Business Horizons 48(2), 143-149 (2005)

14. Crebolder, J., Pronovost, S., Lai, G.: Investigating Virtual Social Networking in the Military Domain. In: 14th International Command and Control Research and Technology Symposium - ICCRTS "C2 and Agility", Washington, DC, June 15-17 (2009)

15. Carrington, P.J., Scott, J., Wasserman, S.: Models and Methods in Social Network Analysis. Canadian Journal of Sociology Online (September-October 2005)

16. Jonnalagadda, S., Peeler, R., Topham, P.: Discovering opinion leaders for medical topics using news articles. Journal of Biomedical Semantics 3, 2 (2012)

17. Hajian, B., White, T.: Modelling inuence in a social network: Metrics and evaluation. In: IEEE Third International Conference on Social Computing (SocialCom) (2011)

18. Bonacich, P.: Factoring and weighting approaches to clique identification. Journal of Mathematical Sociology 2, 113-120 (1972)

19. Freeman, L.C.: A set of measures of centrality based on betweenness. Sociometry 40, 3541 (1977)

20. Bonacich, P.: Factoring and weighting approaches to clique identification. Journal of Mathematical Sociology 2, 113-120 (1972)

21. Kwak, H., Lee, C., Park, H., Moon, S.: Finding influentials based on the temporal order of information adoption in Twitter. In: Proceedings of the 19th International World Wide Web Conference, pp. 1137-1138. ACM (2010)

22. Aus, S., Galuba, W., Huberman, B.A., Romero, D.M.: Influence and passivity in social media. In: ACM Proceedings (2010)

23. Cha, M., Haddadi, H., Benevenuto, F., Gummadi, K.: Measuring user influence in twitter: The million follower fallacy. In: Proceedings of the 4th International Conference on Weblogs and Social Media (2010) 
24. Afrasiabi Rad, A., Benyoucef, M.: Towards Detecting Influential Users in Social Networks. In: Babin, G., Stanoevska-Slabeva, K., Kropf, P. (eds.) MCETECH 2011. LNBIP, vol. 78, pp. 227-240. Springer, Heidelberg (2011)

25. Kempe, D., Kleinberg, J., Tardos, É.: Maximizing the spread of influence through a social network. In: Proceedings of the Ninth ACM SIGKDD International Conference on Knowledge Discovery and Data Mining, pp. 137-146. ACM (2003)

26. Kundu, S., Murthy, C.A., Pal, S.K.: A New Centrality Measure for Influence Maximization in Social Networks. In: Kuznetsov, S.O., Mandal, D.P., Kundu, M.K., Pal, S.K. (eds.) PReMI 2011. LNCS, vol. 6744, pp. 242-247. Springer, Heidelberg (2011)

27. Lerman, K., Ghosh, R., Kang, J.H.: Centrality Metric for Dynamic Networks Analysis. In: Proceedings of KDD Workshop on Mining and Learning with Graphs (MLG), (2010)

28. Juszczyszyn, K., Budka, M., Musial, K.: The Dynamic Structural Patterns of Social Networks Based on Triad Transitions. In: Advances in Social Networks Analysis and Mining (ASONAM) (2011)

29. Braha, D., Bar-Yam, Y.: From Centrality to Temporary Fame: Dynamic Centrality in Complex Networks. Complexity 12(2) (2006)

30. Hill, S.A., Braha, D.: A dynamic model of time-dependent complex networks. Phys. Rev. E 82, 046105 (2010)

31. Wasserman, S., Faust, K.: Social Network Analysis: Methods and Applications. Cambridge University Press, Cambridge (1994)

32. Kleinberg, J.: Authoritative sources in a hyperlinked environment. Journal of the ACM 46(5), 604-632 (1999)

33. Ruhnau, B.: Eigenvector-centrality: a node-centrality? Social Networks 22, 357-365 (2000)

34. Volpentesta, A.P., Felicetti, A.M.: Eigenvector Centrality Based on Shared Research Topics in a Scientific Community. In: Camarinha-Matos, L.M., Boucher, X., Afsarmanesh, H. (eds.) PRO-VE 2010. IFIP AICT, vol. 336, pp. 626-633. Springer, Heidelberg (2010)

35. Kirby, J., Mardsen, P.: Connected marketing: The viral, buzz and word of mouth revolution. Elsevier Ltd (2006) 